\title{
ПРИМЕНЕНИЕ СИНЕКТИЧЕСКОГО ПОДХОДА ПРИ РАССМОТРЕНИИ ТЕХНИЧЕСКОГО ЗАДАНИЯ
}

\section{APPLICATION OF THE SYNECTIC APPROACH WHEN CONSIDERING TECHNICAL REFERENCE}

\section{E. Stepashkina \\ V. Shishkin \\ M. Grishin}

Summary. In this article, the authors of the article proposed a synectic method of the decision-making process using direct analogy during the consideration of the technical task for the development of a new product using the established decision bases of the enterprise structures responsible for the design of the future product, for the development of technological processes, for repair and operation and for financial component. Using electronic structures in the Product Data Management (1C: PDM) system with a complete set of design documentation of previously developed products, the authors select a constructive analogue for its further transformation in accordance with the technical and technological requirements of the customer.

Keywords: theory of inventive problem solving, analogue, product, technical task, product life cycle, synectic approach,.
Степашкина Елена Валерьевна

Аспирант, Ульяновский Государственный

Технический Университет; заместитель начальника серийно-конструкторского отдела, Акционерное общество «Ульяновское конструкторское бюро приборостроения» e.stepashkina85@mail.ru

Шишкин Вадим Викторинович K.т.н., nрофессор, Институт Авиационных Технологий и Управления shvv@ulstu.ru

Гришин Максим Вячеславович

К.т.н., Институт Авиационных Технологий и Управления; инженер-конструктор, Акционерное общество «Ульяновское конструкторское бюро приборостроения» likani7@mail.ru

Аннотация. В данной статье авторами статьи был предложен синектический метод процесса принятия решения с использованием прямой аналогии в ходе рассмотрения технического задания на разработку нового изделия с использованием наработанных баз решений структур предприятия, отвечающих за конструирование будущего изделия, за разработку технологических процессов, за ремонт и эксплуатацию и за финансовую составляющую. Используя электронные структуры в системе Product Data Management (1C: PDM) с полным комплектом КД разработанных ранее изделий, авторы подбирают конструктивный аналог для дальнейшего его преображения согласно технико-технологическим требованиям заказчика.

Ключевые слова: теория решения изобретательских задач, аналог, изделие, техническое задание, жизненный цикл изделия, синектический подход.

\section{Основная часть}

Основанием для начала процесса создания нового изделия является согласованное между заказчиком и исполнителем помимо договора (контракта) и техническое задание (Т3) на разработку, в котором прописывается ход выполнения стадий проектирования конструкторской (КД) и технологической (ТД) документации (полного комплекта КД). Т3 на разработку или модернизацию изделия предполагает совокупность научно-исследовательских (НИР) и опытно-конструкторских работ (ОКР).

Одним из результатов НИР является разработка документа, содержащего требования (совокупность всех показателей, норм, правил и положений) к изделию, его 
изготовлению, контролю, приемке и поставке, которые нецелесообразно указывать в других конструкторских документах [1]. Предприятие, разрабатывающее и/или изготавливающее изделие, документом Технические условия (ТУ) определяют все этапы ЖЦИ, в части производства. На основании предоставленных параметров в ТУ заказчик-потребитель имеет представление о соответствии качественных характеристик, заявленных в Т3. ТУ является совокупностью полного набора требований к изделию, его созданию, контролю производства и приема. ТУ есть не что иное, как совокупность проработанных данных, представленных в Т3, с более четким представлением требований к возможностям и функциям изделия, принципам функционирования, основным параметрами характеристикам, требованиям к сырью, материалам и покупным изделиям, комплектности, маркировки, упаковки, правилам приема, методикам испытаний, методам контроля изготовления, проверкам, хранению и транспортировки.

Согласно ГОСТ 15.016 Т3 на ОКР по разработке нового изделия может состоять из разделов [2]:

- Наименование, шифр ОКР, основание, исполнитель и сроки выполнения ОКР;

- Цель Выполнения ОКР, наименование и обозначение изделия;

- Технические требования к изделию;

- Технико-экономические требования;

- Требования видам обеспечения;

- Требования к сырью, материалам и КИМП;

- Требования к консервации, упаковке и маркировке;

- Требования к учебно-тренировочным средствам (при необходимости);

- Специальные требования;

- Требования к документации;

- Этапы выполнения ОКР;

- Порядок выполнения и приемки этапов ОКР.

Первоначальная стадия «Исследование» ЖЦИ на этапе опытно-конструкторской работы (ОКР) базируется на задаваемых заказчиком ТTТ [5]. Чтобы принять решение о возможности разработки и изготовления предприятию-разработчику необходимо проводить обширный анализ всех имеющихся на рынке изделий и разработанных ранее на предприятии. Таким образом, временные ресурсы предприятия-разработчика направлены на повторное обращение к имеющейся базе аналогов изделий, прорабатывая заданные ТТТ заказчиком на разработку нового изделия. Авторами статьи предлагается автоматизировать возобновляющиеся действия в части исследования путем разработки специализированных средств на основе методов ТРИЗ-технологий с возможностью учета семантики и контролируемого накопления опыта.
Другими словами, на этапе начала бизнес-процесса формирования модели Т3 на проектирование будущего изделия следует использовать пути принятия решения поставленных задач с применением аналогий имеющихся проектных решений [3].

Большинство крупных конструкторских предприятий обладает значительным архивом КД и ТД на изделия. Таким образом, по утверждению авторов статьи, после обращения заказчика с коммерческим предложением, содержащим технико-технологические требования (ТTТ) к работе, деятельность конструкторского предприятия в первую очередь следует направить на поиск ранее разработанных изделий и на анализ технологии возможности изготовления аналога требуемой продукции [3].

Ранее авторами было предложено рассмотреть этапы синектического метода процесса принятия решения (рис. 1) с сопоставлением этапов научно-исследовательской работы в ходе проектирования нового изделия, или модернизации старого. В ходе рассмотрения синектического метода процесса принятия решения на этапах анализа проблематики и генерирования идей авторами было предложено использовать имеющуюся базу на предприятии ранее разработанных и изготовленных аналогов изделий, представляющих собой совокупность технических параметров, несущих в себе базы знаний о проектировании и изготовлении аналога [3].

Основными функциями предприятия-разработчика и предприятия-изготовителя, направленные на организацию процессов создания нового изделия, авторами были выделены [4]:

- анализ потребностей целевой аудитории;

- разработка концепции будущей продукции;

- проектирование и моделирование будущей продукции;

- изготовление, продажа и поставка продукции потребителям;

- послепродажное обслуживание продукции;

- материально-техническое обеспечение производства на предприятии.

Проектирование и моделирование будущей продукции, изготовление, продажа и поставка продукции потребителям и послепродажное обслуживание продукции представляют собой материальную составляющую готового изделия, которая содержит в себе исходные данные для поиска аналога нового изделия с последующим принятием решения для разработки и/или изготовления.

В ходе рассмотрения Т3 на разработку, применяя синектический метод процесса принятия решения с ис- 


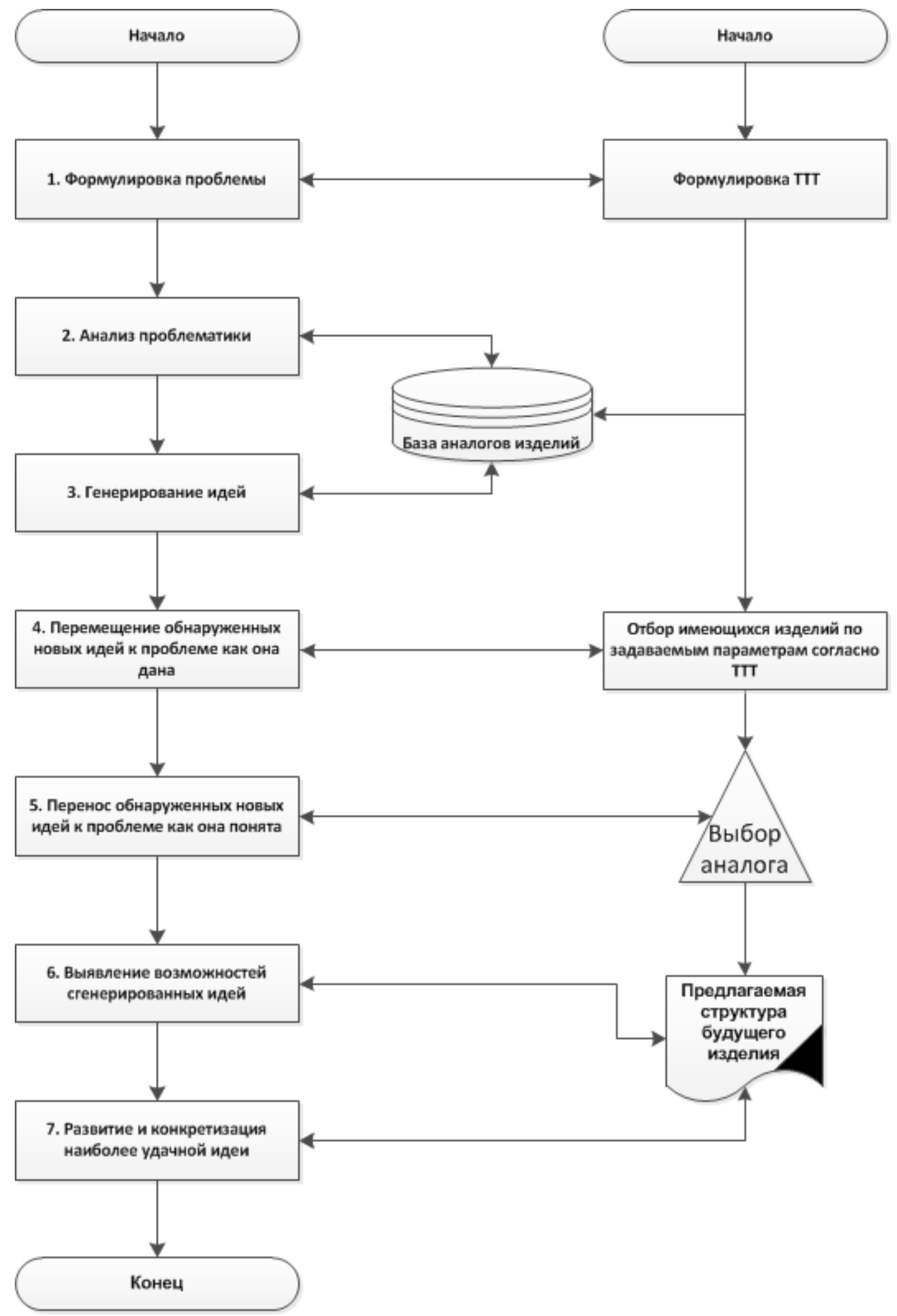

Рис. 1. Этапы синектического метода процесса принятия решения в ходе проектирования будущего изделия 


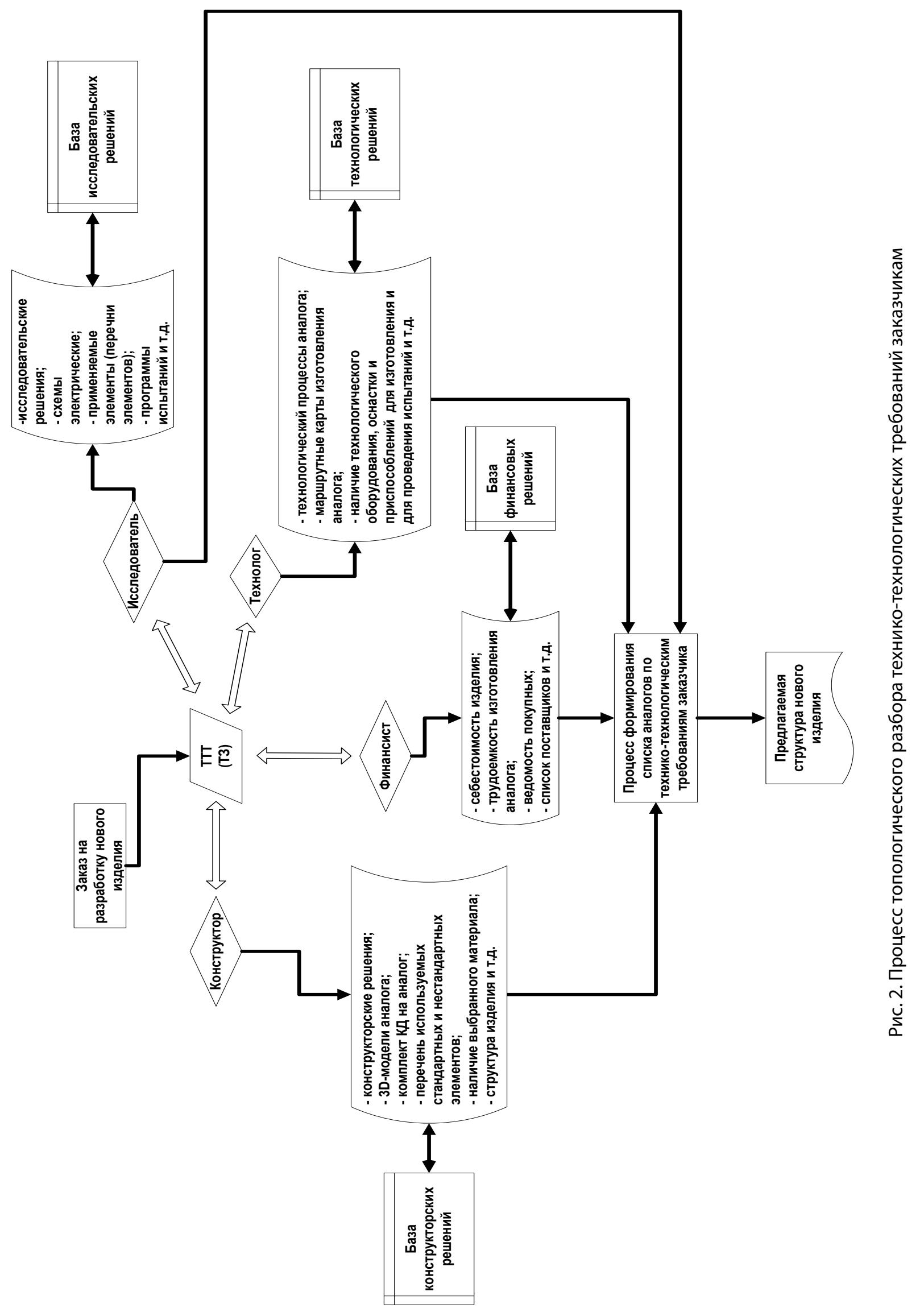




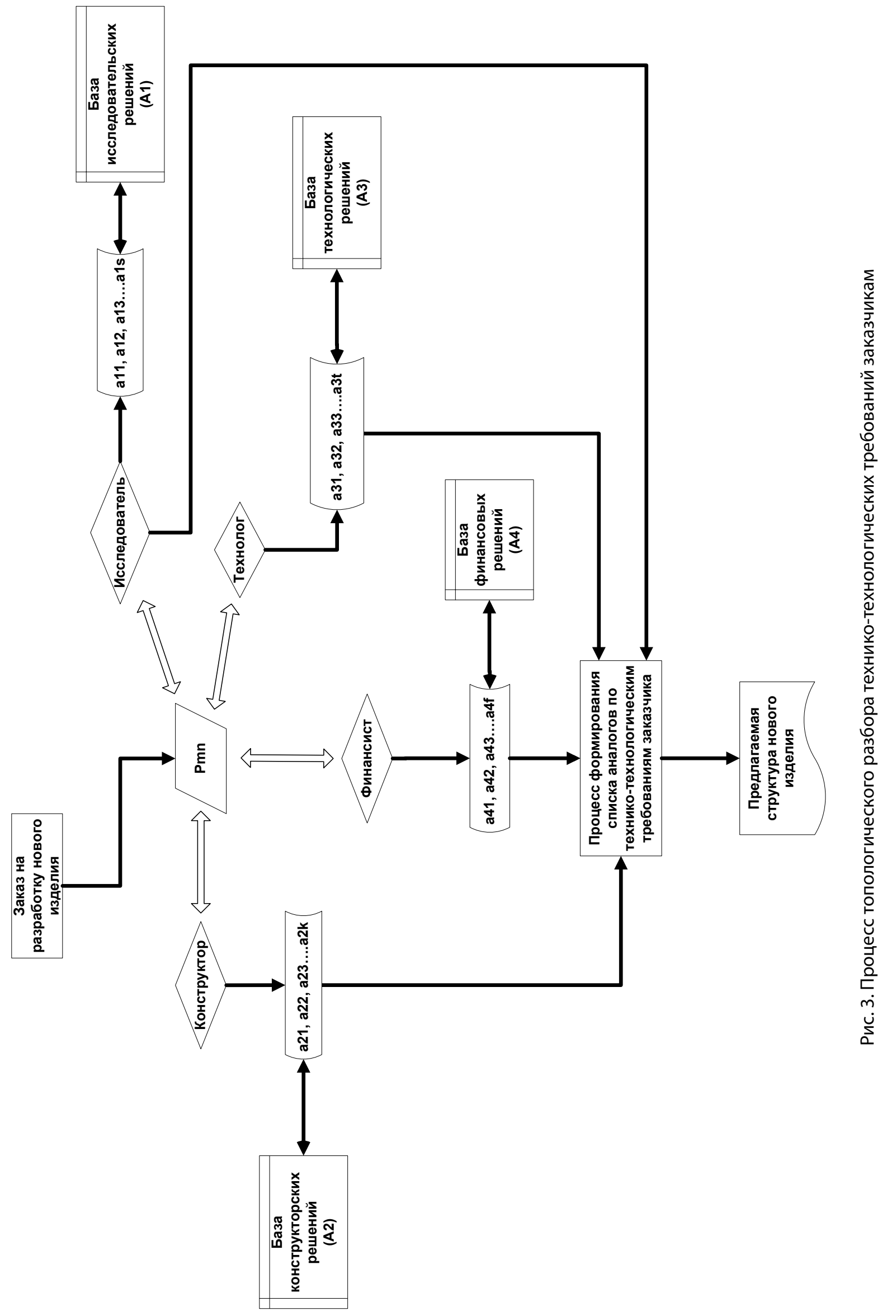




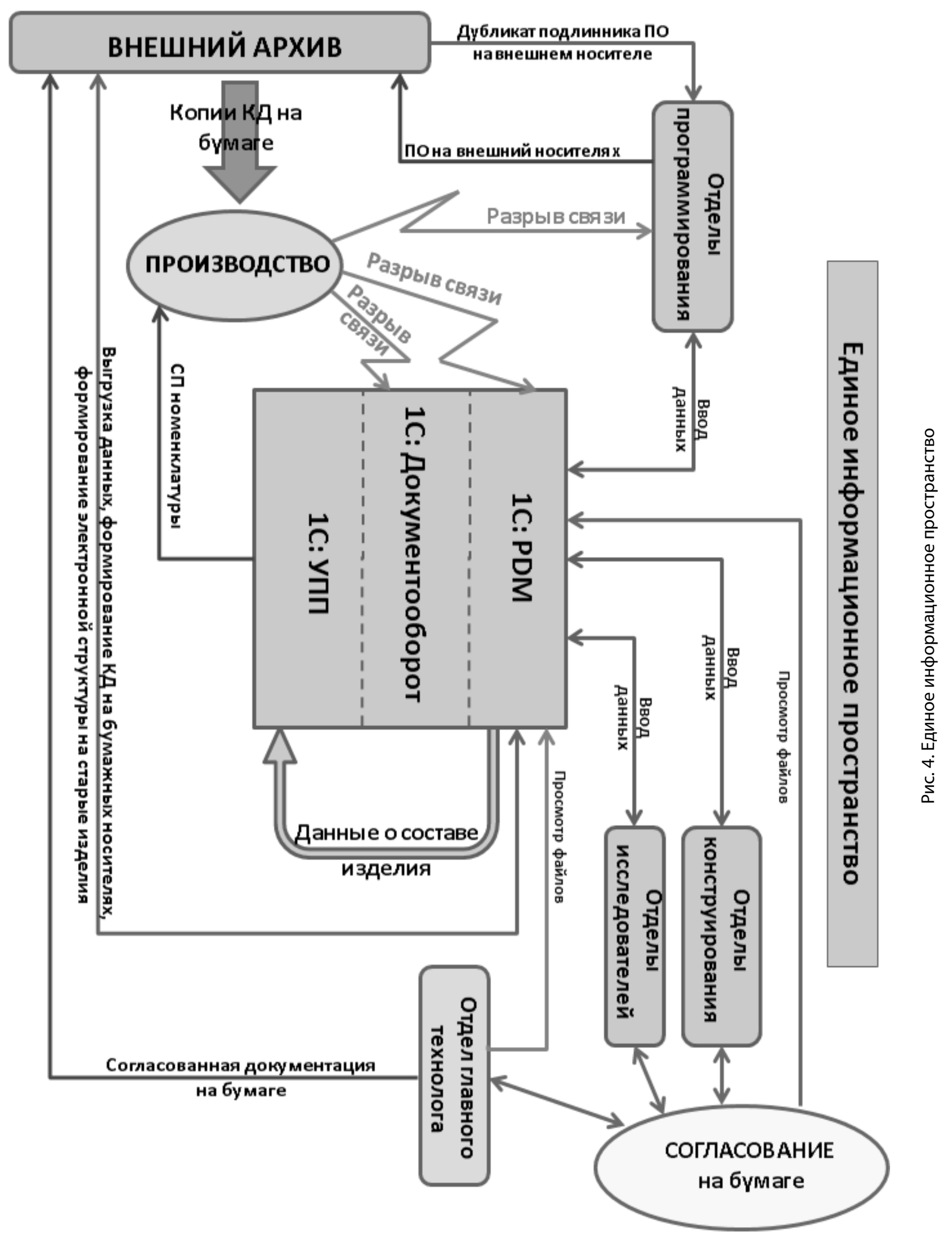




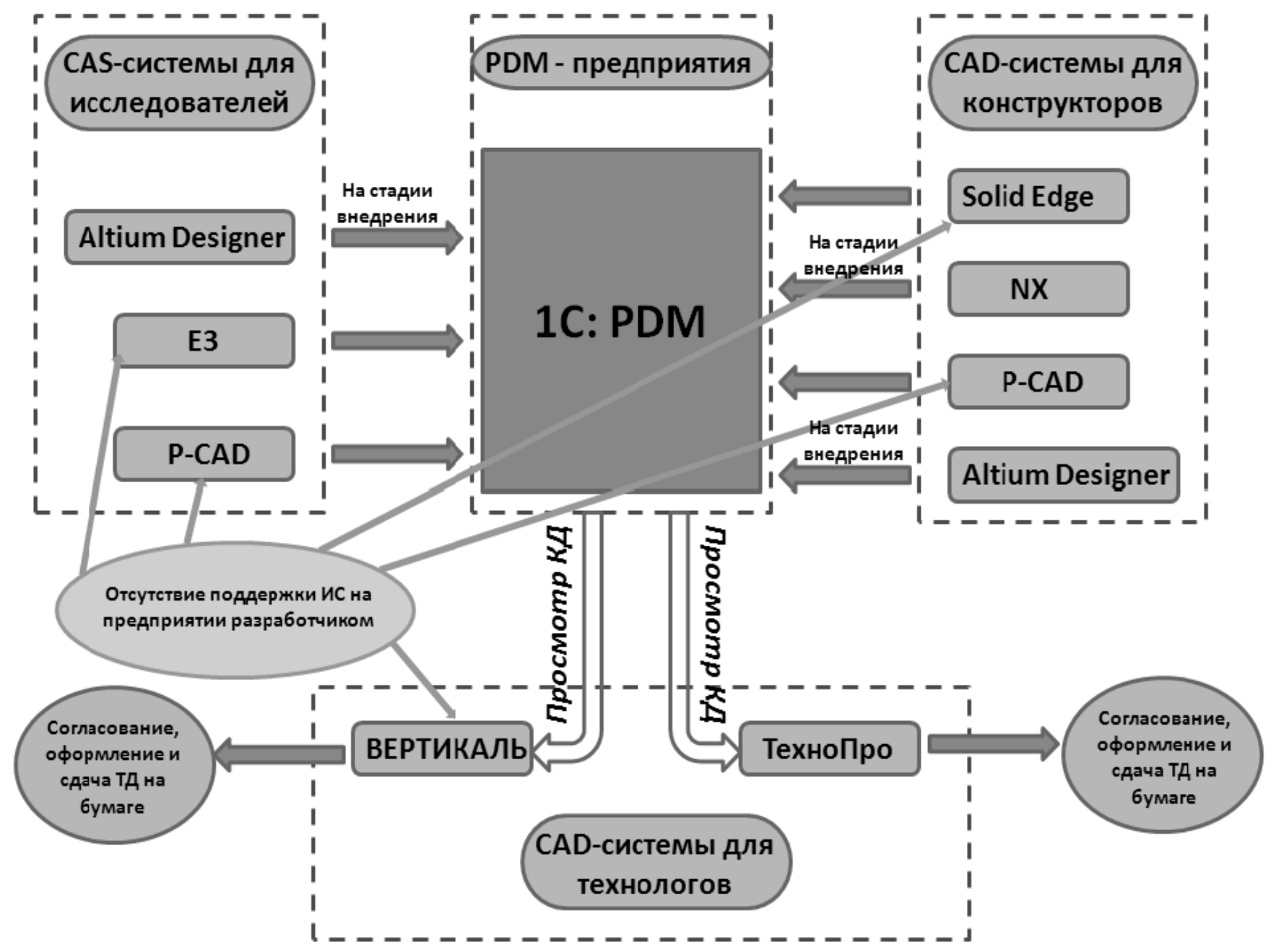

Рис. 5. Применение информационных систем на стадии разработки изделия

пользованием прямой аналогии [4], авторами предлагается задействовать структуры предприятия, отвечающие за конструирование (разработка конструкции) будущего изделия, за разработку технологических процессов, за ремонт и эксплуатацию и за финансовую составляющую.

Рассмотрим этапы 2 «Анализ проблемы» и 3 «Генерирование идей» синектического метода процесса (рис. 1) принятия решения в ходе проектирования будущего изделия, опираясь на задействованные структуры предприятия (рис. 2).

Суть предлагаемого авторами метода синектического подхода принятия решения при рассмотрении ТTТ заказчика заключается в подборе более близкого по своим характеристикам аналога изделия, ранее разрабатываемом и/или изготавливаемом на авиационном предприятии.
При рассмотрении авторами ранее [4] начальные заданные требования $p_{1 n}, p_{2 n}, p_{3 n}, \ldots, p_{m n}$ на изделие путем сравнения имеющихся параметров $p_{1 j}, p_{2 j}, p_{3 j}, \ldots, p_{i j}$ из базы аналогий (подобий) производит подбор ранее разработанной продукции для интеграции цифрового облика изделия для формирования T3 на ОKP.

$$
\begin{aligned}
& P_{m n}=\sum\left(p_{1 n}, p_{2 n}, p_{3 n}, \ldots, p_{m n}\right), m \neq 0, n \neq 0, \\
& P_{i j}=\sum\left(p_{1 j}, p_{2 j}, p_{3 j}, \ldots, p_{i j}\right), i \neq 0, j \neq 0 \\
& p_{m n} \Leftrightarrow p_{i j}, \\
& P_{m n} \Leftrightarrow P_{i j},(4),
\end{aligned}
$$

В свою же очередь каждый параметр ТТТ аналога $p_{i j}$ есть объединение (5) имеющихся решений из сформированных баз $A_{1}, A_{2}, A_{3}, A_{4}$ каждого из ответственно- 
го за разработку и/или изготовление подразделения структуры предприятия-разработчика и/или предприятия-изготовителя, полученное путем синектического отбора.

$$
\begin{aligned}
& p_{i j}=F\left(A_{1}, A_{2}, A_{3}, A_{4}\right), \\
& A_{1}=\sum\left(a_{11}, a_{12}, a_{13}, \ldots, a_{1 s}\right), s \neq 0, \\
& A_{2}=\sum\left(a_{21}, a_{22}, a_{23}, \ldots, a_{2 k}\right), k \neq 0, \\
& A_{3}=\sum\left(a_{31}, a_{32}, a_{33}, \ldots, a_{3 t}\right), t \neq 0, \\
& A_{4}=\sum\left(a_{41}, a_{42}, a_{43}, \ldots, a_{4 f}\right), f \neq 0, \text { где }
\end{aligned}
$$

$A_{1}$ - множество исследовательских решений,

$A_{2}$ - множество конструкторских решений,

$A_{3}$ - множество технологических решений,

$A_{4}$ - множество финансовых решений.

Чем больше совпадений при синектическом отборе, тем определеннее будет предложена структура будущего изделия с дальнейшими изменениями при необходимости (рис. 3).

Если перед предприятием-разработчиком стоит задача в Т3 «разработать» новое изделие, в ходе решения задачи являются основными структуры, отвечающие за исследовательскую и конструкторскую деятельность.
Как писалось ранее [5] в результате цифровизации на ведущем предприятии авиаприборостроительной отрасли с определенным формализмом в управлении компанией и организацией всевозможных бизнес-процессов, было принято решение часть управленческих механизмов перевести на упорядоченную документационную основу. В результате были внедрены ИС (рис. 4):

- 1С: документооборот для решения задачи автоматизации учета документов, взаимодействия сотрудников, контроля и анализа исполнительской дисциплины.

- 1С: ПРЕДПРИЯТИЕ РDМ «Управление инженерными данными» для разработки электронной структуры изделий, оформление конструкторской документации:

- «1С: Управление производственным предприятием» для решения задач управления и учета на производственном предприятии и финансово-хозяйственной деятельности предприятия.

В настоящее время 1C: PDM ведется вся работа конструкторской, исследовательской (рис. 5), связанных с изготовлением новых изделий с использованием других САПР для проектирования.

Таким образом, взяв за основу электронные структуры с полным комплектом КД разработанных ранее изделий, можно подобрать конструктивный аналог для дальнейшего его преображения согласно ТТТ заказчика, что значительно сократит время разработки нового изделия.

\section{ЛИТЕРАТУРА}

1. ГОСТ 2.102-2013 Единая система конструкторской документации. Виды конструкторских документов. М: Изд-во стандартов, 2014. 16 с.

2. Степашкина Е.В., Гришин М.В., Коженков В.А., Алексеев А.А. Взаимосвязь входных и выходных параметров об изделии на стадиях ЖцИ. // Приборы и системы. Управление, контроль, диагностика. 2020. № 4

3. ГОСТ 15.016 <2016 Система разработки и постановки продукции на производство. Техническое задание. Требования к содержанию и оформлению. М: Изд-во стандартов, 2017. 30 C.

4. Степашкина Е.В., Шишккин В.В., Алексеев А.А, Гришин М.В. Применение методов ТРИЗ для анализа технико-технологических требований в ходе формирования технического задания на изделия авиационного назначения в условиях ЖЦ. // Приборы и системы. Управление, контроль, диагностика. 2021. № 4

5. Степашкина Е.В., Горбачев И.В., Гришин М.В. Информационные системы на авиаприборостроительном предприятии // Известия Самарского научного центра РАН, 2019. Т. 21. № 1. С. 128-135

(c) Степашкина Елена Валерьевна ( e.stepashkina85@mail.ru),

Шишкин Вадим Викторинович ( shvv@ulstu.ru ), Гришин Максим Вячеславович (likani7@mail.ru).

Журнал «Современная наука: актуальные проблемы теории и практики» 